\title{
Venous hemodynamics, knowledge and miracles
}

\section{Franceschi ${ }^{1}$}

${ }^{1}$ Centre Marie Thérèse Hôpital Saint Joseph 189 rue Raymond Losserand 75014 Paris

submitted: Sep 28, 2019, accepted: Aug 8, 2020, EPub Ahead of Print: Sep 24, 2020

Conflict of interest: None

DOI: 10.24019/jtavr.83 - Corresponding author:Dr. Claude Franceschi, claude.franceschi@ gmail.com

(C) 2019 Fondazione Vasculab impresa sociale ONLUS. All rights reserved.

\begin{abstract}
The occurrence of the Peregrine's ulcer is scientifically explainable but its miraculous healing in one night cannot be explained by the current scientific knowledge. The long history of fluid mechanics, anatomic and pathophysiologic discoveries led to the better knowledge of hemodynamics in pathophysiology. The last technologies provided strong means to the assessment of venous insufficiency, especially Duplex
\end{abstract}

Ultrasound. Renewed hemodynamic concepts provide a better explanation of the pathophysiology of venous leg ulcers as well as reliable outcomes and less recurrences for varicose veins and ulcers.

Keywords Venous ulcer, Venous hemodynamics, Miracle, CHIVA, Duplex ultrasound

\section{Introduction}

Science denies miracles because it considers them phenomena not yet scientifically elucidated. For believers, a miracle is an event accomplished by a divinity when it is not scientifically explicable. So, the healing of the Peregrine's sore in XIV century was assigned to a divine miracle because at that time it could not be explained by the medical knowledge.

If we refer to the historical data, Peregrine's legs ulcers occurred in the 30th year of a continuous standing posture. This is today explainable by a chronic excess of venous hydrostatic pressure. Six months later he finally fell and laid on the floor and the ulcers healed the successive night. This remains scientifically not understood. Indeed, according to the current scientific knowledge, the venous ulcer healing needs much more than one night stay in recumbent posture.

Aim of the current paper is to give an interpretation of the pathophysiologic phenomena underlying venous ulcers, according to the hemodynamic frame described in the CHIVA therapy of venous diseases. CHIVA is the French acronym for «Cure Hemodynamique de l'Insuffisance Veineuse en Ambulatoire».

\section{Short history of pathophysiology of venous ulcers}

Venous pathophysiology progressed step by step with the discoveries regarding venous anatomy, biology, physiology and fluid mechanics applied to venous hemodynamics.

\section{Fluid mechanics}

Fluid mechanics progressed mainly with Archimedes (c. 287c. 212 BC), Simon Stevin (1548-1620), Blaise Pascal, (1623-1662), Evangelista Torricelli (1608-1647), Isaac Newton ( 1643-1727),
Daniel Bernouilli (1700-1782), Jean-Léonard Marie Poiseuille (17971869). Louis-Marie-Henri Navier (1785-1836), George Gabriel Stokes (1819-1903) and many others.

\section{Hemodynamics and venous pathophysiology}

Ibn Al-Nafis Damishqui (1210-1288) then Giovanni Battista Canano (1515-1579) described the venous valves. William Harvey (1578-1657), pupil of Hieronymus Fabricius, published in 1628 the Exercitatio Anatomica de Motu Cordis et Sanguinis in Animalibus 
where he wrote "Wherefore there are many valves in the veins opposed to the heart: the arteries have none except at the exit from the heart. Hence the first veins are pulsating, the latter are non-pulsating" . He found fierce opponents as Primerose, Reid, Plemp. Guy Patin called him a « circulator » i.e a charlatan in latin" and Jean Riolan sentenced his discovery «paradoxical, useless for medicine, false, impossible, unintelligible, absurd and harmful to the human life». Then Marcello Malpighi discovered the capillaries in 1661. Richard Lower described the vis a tergo (cardiopetal flow provided by the capillaries) and the venarum tonus (venous tone) in 1670, Antonio Valsalva the vis a fronte (cardiac aspiration) in 1710, Justin von Loder the Perforators in 1803 and Chevalier de Richerand the muscular pump in 1817. Ernest Henry Starling (1866-1927) described the Starling's principle ${ }^{1}$, revised then by Levick in $2010^{2}$ : The net flow in each section of the capillary wall (direction and amount of flow) is due to the balance between the pressure difference and the oncotic difference.

\section{Hemodynamics and Treatments}

Sir Benjamin Brodie (1783-1862) wrote in 1846 the "Lecture VIII: Varicose veins and ulcers of the legs" in his book "Lectures illustrative of various subjects in pathology and surgery". He assigned the cause of ulcers to the excessive weight of blood column due the valves incompetence and proposed its treatment by ligation of the greater saphenous vein (GSV) or a tight natural rubber bandaging when the operation was too hazardous. Later, Friederich Trendelenburg (1844-1924) performed the GSV ligation according to his hemodynamic test. When he pressed with his finger the proximal varicose GSV in recumbent posture and kept it pressed when the patient got standing up, the saphenous trunks and their varicose tributaries remained empty. Then, when he removed his finger they dilated instantaneously, because due to the valves incompetence, the weight blood column was maximum, from the cava vein to the distal veins. He hypothesized also a "private circulation" which he described so: "When walking, the blood of the varicose veins is aspirated by the communications when the deep blood is violently pumped upward. Then, probably, part of this deep blood will turn back downwards from the iliac and femoral veins, refilling again the varices". His assistant, Georg Clemens Perthes (1869-1927) described the "Perthes test" which confirmed his intuition. Indeed, the manoeuvre showed that when the patient walks with a tight tourniquet tied at the thigh, the varicose vein emptied or not according to the patency of the deep venous veins. Nevertheless, despite the efficiency of GSV ligation as a cure of the venous ulcers in the "safe in the skillful hands of Trendelenburg", this operation was not widespread because too dangerous and sometimes deadly (previously pointed out by Benjamin Brodie). Then, the progresses in antisepsy and anesthesia made possible and safer the surgical ligation. Moreover, the ligation was replaced by the radical ablation of the GSV (stripping by Keller (1905), Mayo (1906) and Babcok (1907).

Unfortunately, this radical stripping reduced the venous hemodynamics to a simplistic concept. Indeed, the varicose veins were regarded as the cause of the venous insufficiency instead of being considered themselves victims of the same hemodynamic impairement. Consequentely the recurrence was assigned to an incomplete venous eradication. So "the more the ablated varicose veins, the best the outcomes and the less the recurrence" was and still is for many the dogma. The stripping-based concepts led to alternative ablative procedures which consisted on endo-venous injections of various occlusive products. Jean Sicard (1920), Karl Linser, Raymond Tournay (1893 -1984) used products less dangerous than the previous injected by Valette, Petrequin, Desgranges (1853) and Weinlechner (1884).

\section{TRANS-MURAL Pressure (TMP)}

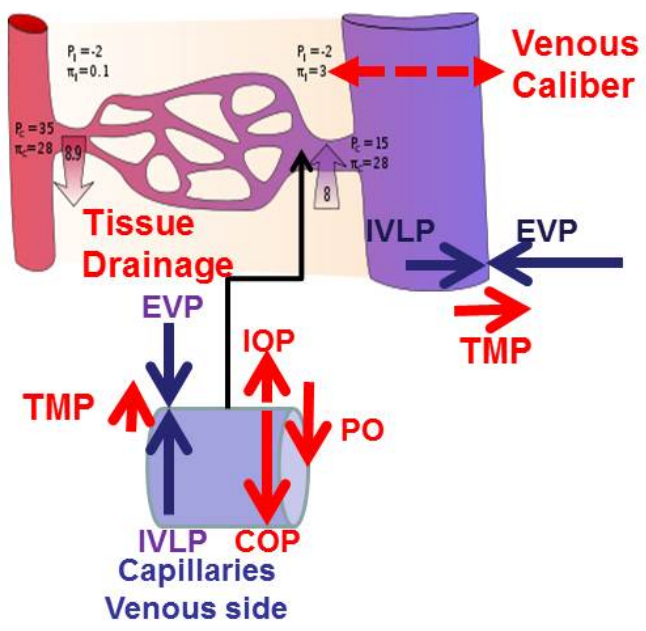

Figure 1 - Action of the Trans-mural pressure and the oncotic pressure on tissue drainage and venous calibre. TMP - Trans-mural pressure, EVP - Extra-venous Pressure. Po - oncotic pressure. IOP - Interstitial oncotic pressure. PO Resultant oncotic pressure. IVLP - Intra-venous lateral pressure. 


\section{Current state of venous hemodynamic knowledge \\ Ablative approach}

Duplex Ultrasound investigation extended this old ablation dogma to all the visible perforators and to any refluxing vein, dilated or not, and favoured the explosion of endo-venous of still more "innovative" procedures (SEPS, LASER, Trivex, Foam, RF, Glue ....). Thus, these new techniques provide new support to a one-century-old concept. Even worse, they destroy more and more easily the lifesaving GSV. Indeed, since the first venous by-pass in 1948 by Jean Kunlin ${ }^{3}$, the competent or incompetent GSV remains the best grafting material ${ }^{4,5}$. The destructive rational, based on the association "reflux-bad vein" is misleading because this "reflux" is defined univocally, whatever its hemodynamic and pathogenic significance according to the pressure, the content, the origin and the destination of the flow. In addition, it is demonstrated that venous flow causes inflammation not according to its direction, but to its pressure/volume amount ${ }^{6}$. Surprisingly, these arguments are still overlooked by the ablative methods. In addition, most procedures include also the ablation of all perforators while most of them have a draining function ${ }^{7-10}$.

\section{The Hemodynamic and conservative approach}

\section{Basic fluid mechanics rules}

Basic hemodynamic parameters and rules can be summarised as below (Fig. 1):

$$
\text { TMP = IVLP - EVP; }
$$

where TMP: Trans-Mural Pressure ; IVLP: IntraVenous Lateral Pressure ; EVP: Extra-Venous Pressure.

$$
\text { IVLP = GHP + LRP + LVMPP; }
$$

where GHP: Gravitational Hydrostatic Pressure; RP: Residual Pressure; LRP: Lateral residual pressure or Lateral part of RP; VMPP: Valvo-muscular pump Pressure; LVMPP: Lateral part of the VMPP.

$$
\mathrm{EVP}=\mathrm{TP}+\mathrm{IFP}+\mathrm{AtP}
$$

where TP: Tissue Pressure, generally exerted on the tissues by the surrounding structures or externally; IFP: Interstitial Fluid Pressure; AtP: Atmospheric Pressure.

Thus, unifying the above formulas

$$
\begin{aligned}
& \mathrm{TMP}=\mathrm{IVLP}-\mathrm{EVP}=(\mathrm{GHP}+\mathrm{LRP}+\mathrm{LVMPP})-(\mathrm{TP} \\
& +\mathrm{IFP}+\mathrm{AtP})
\end{aligned}
$$

The Stevin law sets the value of the GHP

$$
\mathrm{GHP}=\rho g h ;
$$

where $\rho$ is the volumetric mass density, $g$ the gravitational acceleration $\left(\mathrm{m} / \mathrm{s}^{2}\right)$ and $h$ the height of the liquid column.
Hemodynamic and conservative based treatments, are not only represented by leg elevation which decreases the Gravitational Hydrostatic Pressure (GHP) in proportion to the height of the leg elevation, but also by all means which increase the Extra-Venous Pressure (EVP) as bandaging, stockings, immersion into-liquids and pneumatic compression.

The CHIVA Cure ${ }^{7,8}$ was proposed in 1988 to improve the conservative and hemodynamic approach by means of a minimally invasive surgery. Its hemodynamic fundamentals revive and synthetize in a whole hemodynamic model the venous pathophysiology. So, it took profit of the past hydrodynamic, pathophysiological and clinical discoveries and studies up to Bjordal ${ }^{11}$ and the dynamic EchoDoppler investigations $^{12,13}$. It considers the venous system as the whole of pumps (right heart, thoraco-abdominal pump, valvo-muscular pumps), conduits (venules and veins) and draining units (capillaries). Its basic definition of the venous insufficiency is, whatever its signs and symptoms, the incapacity of the venous system to provide a proper Trans-Mural Pressure (TMP) to insure a correct function of drainage, right heart ventricle pre-load and thermo-regulation. The excess of TMP is responsible of varicose veins, tissue drainage impairment and it's ulcerous consequence ${ }^{9,10}$.

The lateral pressure (LP) is the sum of the gravitational (GHP) and static (p) pressure of the Bernoulli equation $\left(1 / 2 \rho v^{2}+\rho g h+p=\right.$ Constant, $p=$ static pressure, $1 / 2 \rho v^{2}$ is the dynamic part of the energy, $v=$ velocity).

An increase in fluid velocity occurs simultaneously with a decrease in pressure or a decrease in the fluid's potential energy (or both).

The Poiseuille equation sets the relationship between pressure, flow and resistance in a hydraulic circuit:

$$
\Delta \mathrm{p}=8 \mathrm{~L} \mu \mathrm{Q} / \pi \mathrm{r}^{4}
$$

where $\Delta \mathrm{p}$ is the pressure difference between the two terminals, $L$ is the length of pipe, $\mu$ is the dynamic viscosity, $r$ is the radius of the pipe and $Q$ the volume flow.

The term $8 L \mu Q / \pi r^{4}$ is the resistance $\mathrm{R}$ to the flow.

The residual pressure (RP) is what remains of the arterial pressure downstream the pressure drop due to the resistance of micro-vessels and capillaries.

ST: Shear stress is the amount of the tangential force per unit area of the inner venous wall.

OP: Osmotic Pressure: the outwards lateral pressure which forces out the fluids through the pores of the capillaries is opposed by the inwards oncotic pressure difference between the interstitial fluids and the capillary plasma. 


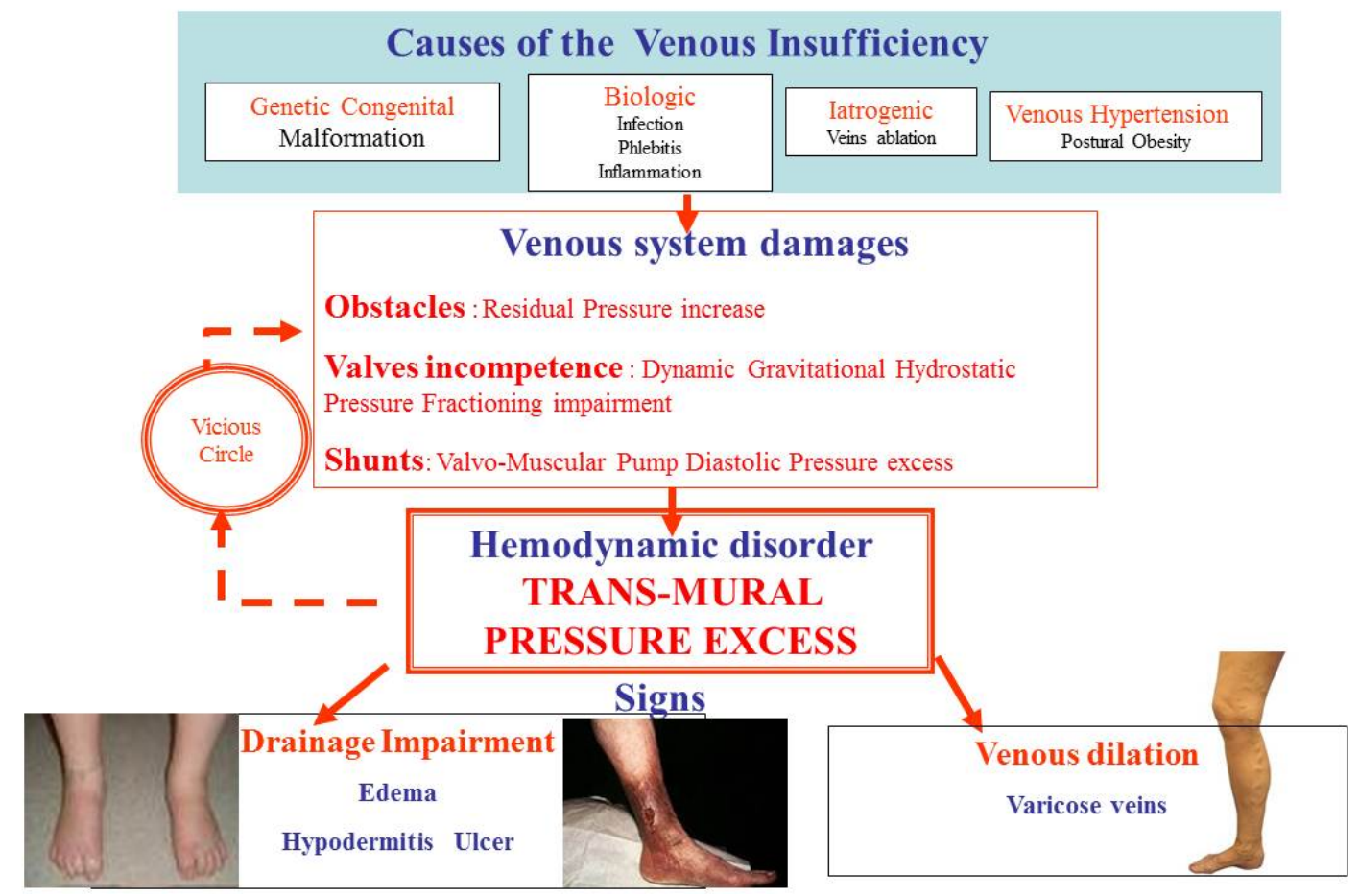

Figure 2 - Causes and effects of the excess of Trans-mural Pressure.

\section{Hemodynamics of venous pathophysiology}

Fig. 2 The hemodynamic treatment of the signs and symptoms of the venous insufficiency proposed by CHIVA aims to restore to normal the excessive TMP.

\section{Excess of IVLP parameters}

LRP (Lateral Residual pressure) increased by obstacles to the outflow (Bernoulli's dynamic-static conversion and accumulation of energy provided by arterial pressure throughout the micro-circulation). It can be naturally compensated by collaterals (Open Vicarious Shunts OVS), surgical by-pass, or unblocked and stented. Thus, the hemodynamic effect of obstacles should be assessed by venous pressure measurements (Catheter or Doppler), because sometimes the natural collateral compensation is sufficient to normalise the RP so making any treatment useless.

As the veins destruction/ablation impairs the tissue drainage and produces varicose recurrence due to the LRP increase, CHIVA preserves the varicose veins even if refluxing. LRP can also increase by an excessive inflow due to micro-circulation vasodilation (heat, inflammation) or arterio-venous fistulae. GHP is excessive when the blood column is not segmented because all the valves keep open in standing and sitting immobile postures and becomes pathogenic when these postures last too long (the Peregrine's case). It is dramatically reduced by walking, thanks to the Dynamic Fractioning of the Gravitational Pressure (DFGP) (systo-diastolic alternate closure of distal and proximal valves of the valvo-muscular pumps VMP). It isn't reduced instead in proportion to missing valves or to their incompetence. Thus, the hemodynamic treatment will fraction the blood column (Trendelenburg strategy and part of CHIVA). Notice that standing still the venous pressure at the foot is not equal to the height head-foot of the venous blood column but to the xyphoid-foot height.
This discrepancy can be explained by hydrostatic laws. The head and thoracic pressure is negative due to the osseous shield which opposes the transmission of the external atmospheric pressure ${ }^{14}$.

LVMPP (Lateral Valvo-muscular pump Pressure) excess. The VMP systole closes the upstream (DFGP) and open the downstream valves, propelling a volume /energy absorbed by the heart aspiration. In case of downstream obstacle, the pressure/volume is no more properly absorbed. That increases the IVLP and causes the Venous claudication. Later, RP can decrease when the resistance is reduced by the progressive opening of the collaterals (Open Vicarious Shunt OVS). The VMP diastole aspires the upstream flow and closes the downstream valves which blocks the reflux and fractions the GHP. In case of valve incompetence, the systolic energy turns backwards during the diastole, increases the distal IVLP and impairs the DFGP (Dynamic Fractioning of the GHP).

When the diastolic reflux overloads an incompetent superficial vein as the GSV we call it a Venous Closed Shunt (CS) where the saphenous femoral junction (SFJ) represents the Escape Point (EP) and the distal perforator the Re-entry Point (R-P). The proximal refluxing and overloading deep-superficial connection are the Escape Point EP and the distal superficial-deep inwards connection is the Reentry Point R-P. The progressive dilation of the veins and varicosities is due to this repetitive CS diastolic overload and its consequent Shear Stress ST. So, walking paradoxically increases the varicose calibre. CS is a closed circuit which resembles the "private circulation" foreseen by Trendelenburg. It differs because Trendelenburg suspected its activation by the VMP systole "varicose veins is aspirated by the communications when the deep blood is violently pumped upwards" while the CS is activated by the diastole. Indeed, the Dynamic Tests 
as the Paraná manoeuvre ${ }^{11}$ show the diastolic emptying of the varicose veins achieved by the muscular diastolic aspiration.

So, another pillar of the CHIVA strategy will consist in disconnecting the CS at the very EP in order to suppress the volume/ energy overload of the varicose vein, leading to a normal EVP and function whilst the flow remains opposite to the "normal" direction (reflux). It improves the drainage of the skin, collapses to normal the vein's calibre (even if previously varicose) and heals the venous ulcers $^{15}$.

The Perthes test is strongly suggested to foresee the effects of CHIVA. Indeed, it shows the expected grade of post CHIVA varicose collapse when a tourniquet is placed at the same site as the planned disconnections. In addition, it can diagnose varicose veins due to a

\section{Hemodynamics of CHIVA strategy}

\section{Four pillars}

- $\quad$ 1-Division of the blood column ${ }^{16}$

2-Disconnection of the veno-venous shunts (Closed Shunts CS and Open Deviated Shunts ODS) at the very source of the diastolic overloading flows called escape points: SFJ, saphenous popliteal junction (SPJ), Pelvic escape outflows, refluxing perforators, tributaries connections to the GSV and SSV

- 3- No extensive phlebectomy of the varicose tributaries in order to respect the drainage of the skin and prevent compensatory recurrence.

4-Preservation of the GSV to prevent recurrence and save a potential vital graft.

\section{Causes, diagnosis and treatments of the venous ulcers}

\section{Causes}

We can rationally assume that TMP excess of the capillaries weakens the skin because it hampers the drainage of the toxic catabolites which triggers inflammation and necrosis. It can be due to a venous obstruction (Residual Pressure excess), a missing DFGHP in case of venous valves incompetence or a too long immobile standing. Despite a healthy venous system, a chronic excess of GHP due to very long immobile standing, could be sufficient to explain the Peregrine's case. It can also explain the prevalence of the venous ulcers in people who works in the standing position. Nevertheless, these people often show additional bad conditions as obesity (Thoraco-abdominal pump impairment and inferior vena cava compression). In addition, the chronic postural blood stasis can damage the venous valves (stasis inflammation) and cause a secondary incompetence.

The ulcer location at the ankle may be explained by the poor vascularisation and thickness of sub-cutaneous tissues of malleolar skin. When the ulcer is connected to a large perforator, the perforator was always considered the cause of a systolic thrust. This believing led to the systematic ablation of all the perforators ( Linton, Foam, LASER, SEPS) However, in the majority of the venous ulcers, the cause is a superficial reflux of a CS and this perforator is a Re-entry point $(\mathrm{R}$ P), "unique" way of drainage of the ulcer unfortunately overload by the diastolic overflow and the excessive blood column height. Thus, deep venous impairment. CS exist also in the deep veins and their disconnection can be proposed according to the deep CHIVA strategy (CHIVP). Sometimes, the Perthes test doesn't empty the varicose veins. This can be due to a deep venous block or a severe valve incompetence. In case of blocked deep veins, the systolic antegrade flow of the varicose veins must be respected because it is compensatory (Open Vicarious Shunt or OVS). In case of deep venous incompetence, the pressure of the deep venous diastolic reflux can be high enough to impede the superficial diastolic re-entering flow. We called it a "deep competitive reflux".

The reflux doesn't activate inflammation because of its retrograde direction, but because of its volume/pressure amount. Indeed, the ablation of the overloading flow improves the inflammatory phenotype of a reflux ${ }^{6}$.

disconnecting the shunt and fractioning the blood column ablates the overload and respects the drainage which results in the ulcer healing ${ }^{17}$. In fewer cases this thrust could exist when the perforator is a systolic escape point of an OVS or a diastolic escape point of a deep reflux.

The ulcer can occur spontaneously or can be triggered by a trauma. Inflammation and necrosis overload the lymphatics so favouring the secondary infection which enlarges the ulcer area.

\section{Diagnosis}

Clinically the ankle location of the ulcer combined with dermitis, varicose veins and edema is a hint to a venous cause but very often the specific hemodynamic cause cannot be found. Thus, an accurate assessment of the venous system with Duplex Ultrasound is mandatory to provide an extensive hemodynamic cartography of the obstructions, refluxes, superficial and deep shunts ${ }^{16}$. The venous pressure measurement, invasive or non-invasive (Doppler measurement of the Posterior tibia vein (PTV) at the ankle in the lying position ${ }^{17}$ ), plethysmography, can assess the respective responsibility of obstruction or venous incompetence for the TMP excess ${ }^{18,19}$.

In our experience, the combination of the venous insufficiency with the ischemic arteriopathy is not a cause of "mixed ulcers", but independently of venous and/or arterial ulcers according to their location. In that case, the potential need of arterial by-pass justifies more than ever the GSV conservative treatment.

\section{Treatment}

\section{The TMP correction is the core of the treatment.}

IVLP reduction is achieved by leg elevation, hemodynamic blocks by-pass, unblocking or stenting, CHIVA and CHIVP (CHIVA procedure applied to deep venous incompetence) according to the hemodynamic configuration. CHIVA and CHIVP ${ }^{20-22}$ consist of

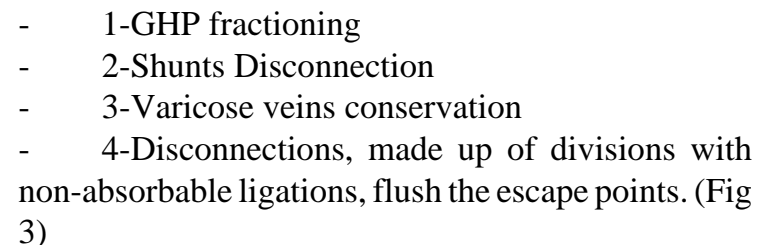
3)

Extra-venous Pressure (EVP) is increased by bandaging and stockings. We prefer inelastic bandaging because less risky of ischemia 
in recumbent posture and more compressive when walking because of its superior efficacy on the venous pumping function ${ }^{23}$. In order to prevent its compressive pressure lowering due to the oedema reduction along the day, we anticipate its shrinking. For that, we treat the patient in recumbent position for 2 hours with the legs elevated and placed on a high stand with a light elastic compression, in order to reduce dramatically the oedema. Then the elastic bandage is removed and the inelastic bandaging is performed. The safe arterial vascularisation is checked with a Doppler assessment of the inter-metatarsal flow. Such position is advised at home using a simple large suit case as stand. The bandaging turnover is one week. (Fig 4)

Local dressings are supposed to clean and treat the secondary infection of the ulcer in order to favour its healing. We use since decades a mixture Vaseline $40 \%$ - Sugar $60 \%$ (Granulated, Icing or Glucose) whatever the dampness and the exudate, without any debridement nor any additive antibiotic or antiseptic, at the same time as the bandaging. The bad smelling disappears, the ulcer necrotic tissue is removed by a simple wiping and the sterilisation is achieved within one or two weeks $^{24}$

\section{Discussion}

The story of the venous ulcer and its hemodynamic relation cannot be exhaustive due to the asynchrony and geographical scattering of the discoveries regarding the fluid dynamics, the anatomy and the pathophysiology of the venous system. Still today, controversy and discrepancy keep going in the phlebologist's world. So, we reduced this paper to our experience and to our and other studies that support our current stand point ${ }^{15,24-28}$.

\section{Conclusion}

The occurrence of the Peregrine's ulcer is scientifically explainable but its miraculous healing in one night is not yet explained by the current scientific knowledge.

Nowadays the efficacy of the treatment results from a long story of the combination of fluid mechanics, anatomic and pathophysiologic discoveries which improved the treatment of the venous ulcers. This is mainly due to the better knowledge of the hemodynamic aspect of the pathophysiology ${ }^{7-10,15}$ and to the strong advancements in technology, particularly the Duplex Ultrasound. Unfortunately, very often technological innovations are not themselves valuable advancements in the comprehension of pathophysiology.
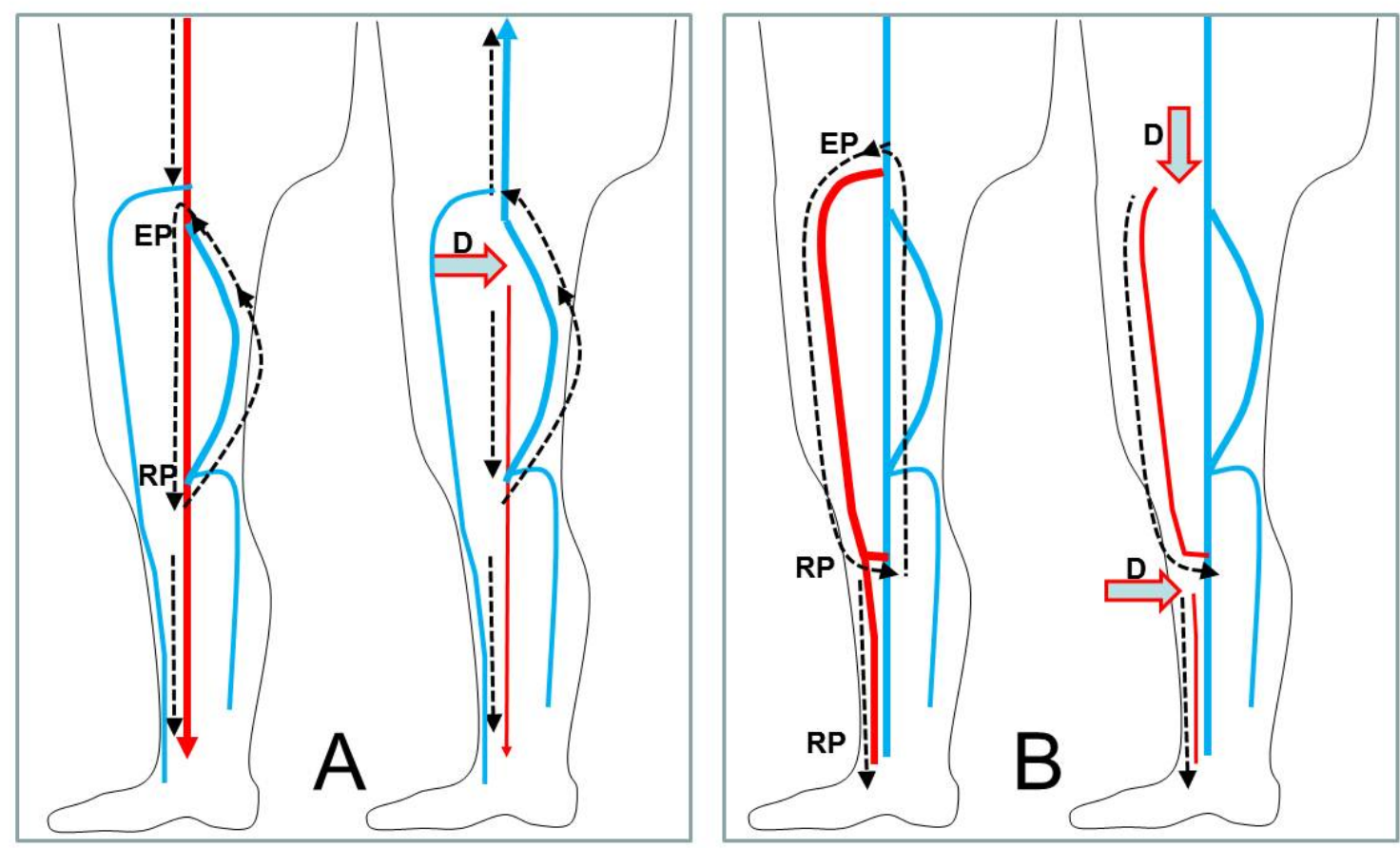

Figure 3 - CHIVA strategy. Examples.

A: Deep closed shunt. Deep venous Ilio-popliteal reflux and competent deep femoral vein. Deep closed shunt: EP: diastolic Escape Point Deep femoral vein-Femoral vein. RP : Re-entry Point. D Closed shunt disconnection + GHP fractioning. B: Superficial closed shunt. GSV reflux : EP - diastolic Escape Point Femoral vein-GSV. R-P - Re-entry Points . D Closed shunt disconnections + GHP fractioning 


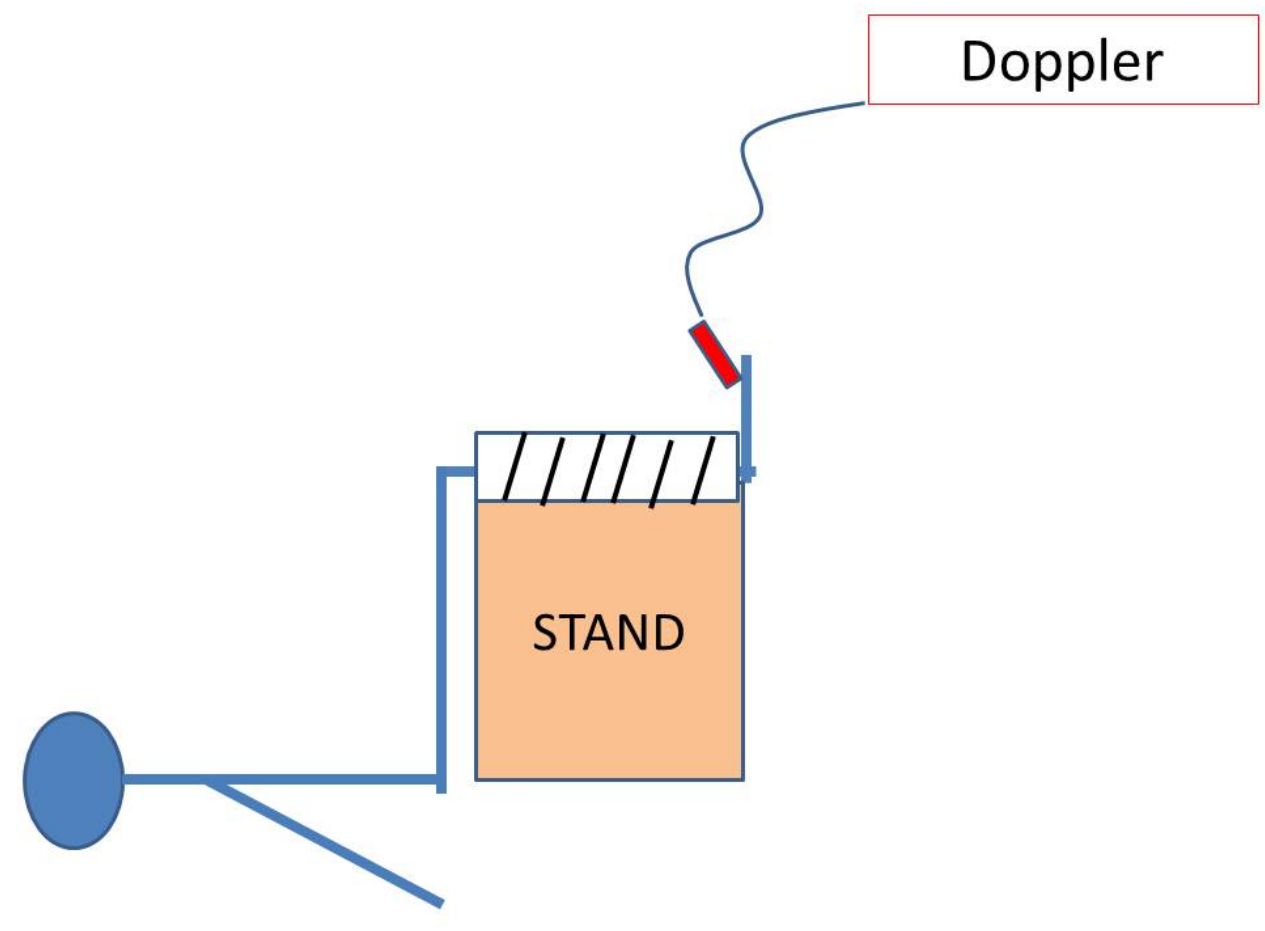

Figure 4 - Draining posture for 2 hours + elastic compression. Then the elastic compression is replaced by an inelastic bandage. The fore foot arterial flow is checked by Doppler.

\section{References}

1) Starling EH. On the absorption of fluids from the connective tissue spaces, J Physiol. 1896, vol. 19 (pg. 312-326)

2) Levick JR, Michel CC. Microvascular fluid exchange and the revised Starling principle. Cardiovasc Res. 2010 Jul 15;87(2):198-210. doi: 10.1093/cvr/cvq062. Epub 2010 Mar 3.

3) Leriche R, Kunlin J. Possibilité de greffe veineuse de grande dimension $(15$ à $47 \mathrm{~cm})$ dans les thromboses artérielles étendues. [Possibility of large venous grafting (15 to $47 \mathrm{~cm})$ in extensive arterial thrombosis] C. R. Académie des Sciences 1948 ; 227 :939-940

4) Romiti M, Albers M and al. Meta-analysis of infrapopliteal angioplasty for chronic critical limb ischemia. J Vasc Surg 2008; 47:975-81.

5) Johansson B, Samano $\mathrm{N}$ and al . The no-touch vein graft for coronary artery bypass surgery preserves the left ventricular ejection fraction at 16 years postoperatively: long-term data from a longitudinal randomised trial. Open Heart 2015 Mar 24;2(1):e000204. doi: 10.1136/ openhrt-2014-000204 eCollection 2015.

6) Zamboni $\mathrm{P}$ et al. Oscillatory flow suppression improves inflammation in chronic venous disease. journal of surgical research 2016; 205:238-245

7) Franceschi C. Theorie et pratique de la cure Conservatrice et Hémodynamique de l'Insuffisance Veineuses en Ambulatoire. [Theory and practice of the Conservative Hemodynamic cure of Incompetent and Varicose veins in Ambulatory patients] Editions de l'Armançon. Précy-sousThil, 1988. http://angio.info/CHIVA_livre_1988\%20Fran\%c3\%a7ais \%2032\%20Mhz\%20.pdf, http://angio.info/CHIVA Book English \%201988-1993\%2026Mhz\%20.pdf
8) Franceschi C. La cure hémodynamique de l'insuffisance veineuse en ambulatoire (CHIVA). [Conservative an hemodynamic treatment of venous insufficiency in outpatients]. J Mal Vasc. 1992;17(4):291-300.

9) Franceschi C. Dynamic fractionizing of hydrostatic pressure, closed and open shunts, vicarious, varicose evolution: how these concepts made the treatment of varices evolve? Phlebologie 2003;56(1):61-66.

10) Franceschi C, Zamboni P. Principles of Venous Hemodynamics. Novapublishers.com. New York, 2010

11) Bjordal R. Simultaneous pressure and flow recordings in varicose veins of the lower extremity. Acta Chir Scand 1970; 136:309-317.

12) Franceschi C. L'Investigation vasculaire par ultrasonographie Doppler. Masson Editeur. Paris, 1977.

13) Franceschi et al. Précis d'échotomographie vasculaire. [A primer of vascular echotomography] Vigot Editeur, Paris, 1986

14) Franceschi C. Paradoxical ankle venous pressure in standing and walking compared to the venous blood column height. JTAVR 2017;31-36. DOI: $10.24019 /$ jtavr.24

15) Zamboni $P$ et al. Minimally invasive surgical management of primary venous ulcers vs. compression treatment: a randomized clinical trial. Eur J Vasc Endovasc Surg. 2003 Apr;25(4):313-8. doi: 10.1053/ejvs.2002.1871

16) Franceschi C, Cappelli M, Ermini S, Gianesini S, Mendoza E, Passariello F, et al. CHIVA: hemodynamic concept, strategy and results. Int Angiol 2016;35:8-30. 
C Franceschi - Venous hemodynamics, knowledge and miracles

17) Bartolo $M$, Antignani PL, Nicosia PM, Todini AR. Noninvasive venous pressure measurement and its validation. Int Angiol $1988 ; 7: 182-9$.

18) Laaengh Massoni $C$, Franceschi $C$. Bilan hémodynamique de la maladie post phlébitique.[Hemodynamic assessment of postthrombotic syndrome] J Mal Vasc. 2008;01.015

19) Franceschi C. Hémodynamique de la maladie postphlébitique : conséquences diagnostiques et thérapeutiques. [Hemodynamics of post-thrombotic syndrome: diagnostic and therapeutic consequences] DOI: 10.1016/j.jmv.2008.01.014

20) Tazi O, Petit M.D, Lazareth I, Franceschi C. Traitement chirurgical hémodynamique des ulcères veineux rebelles.. [Hemodynamic surgical treatment of resistant venous ulcers] jmv.2008.01.017

21) Maeso J, Juan J, Escribano JM, Fernandez Valenzula V, Mestres G, Matas M. Traitement du syndrome post-phlébitique par résection partielle de la veine fémorale superficielle. [Treatment of post-phlebitis syndrome by partial resection of the superficial femoral vein] 10.1016/j.jmv.2008.01.016.

22) Franceschi C. Vous connaissez la CHIVA ? Et connaissezvous la CHIVP. [Do you know the CHIVA ? and the CHIVP too ?] Controversies \& updates actualities in Vascular Surgery. Paris.2018;
Video https://www.youtube.com/watch?v=Ktdrv7RO3FE\&t=10s. Last accessed on Sep 28, 2019.

23) Mosti G, Partsch H. Inelastic bandages maintain their hemodynamic effectiveness over time despite significant pressure loss. J Vasc Surg 2010;52(4):925e31.

24) FranceschiC, Bricchi M, Delfrate R. Anti-infective effects of sugar-vaseline mixture on leg ulcers Veins and Lymphatics 2017; $6: 6652$

25) Carandina S, Mari C, De Palma M, 'et al. Varicose vein stripping vs haemodynamic correction (CHIVA): a long term randomised trial. Eur J Vasc Endovasc Surg 2008; 35, 230-7

26) Parés J, Juan J, Tellez R, et al. Stripping Versus the CHIVA Method: A Randomized Controlled Trial, Annals of Surgery 2010;251:624-631

27) Bellmunt-Montoya S, Escribano JM, Dilme J, MartinezZapata MJ. CHIVA method for the treatment of chronic venous insufficiency. Cochrane database Syst Rev. 2013;7(7):CD009648. 10.1002/14651858.CD009648.pub2.

28) Guo et al. Long-term efficacy of different procedures for treatment of varicose veins. A network meta-analysis. Medicine 2019;98:7 